\title{
SoMe and Self Harm: The use of social media in depressed and suicidal youth
}

\author{
Jennifer L. Carey \\ Emergency Medicine and Medical Toxicology \\ University of Massachusetts Medical School \\ Jennifer.Carey@umassmemorial.org \\ Brittany Chapman \\ Medical Toxicology \\ University of Massachusetts Medical School \\ brittany.chapman@umassmed.edu \\ Peter R. Chai \\ Emergency Medicine \\ Brigham and Women's Hospital \\ pchai@bwh.harvard.edu \\ Danielle E. Jake-Schoffman \\ Division of Preventive and Behavioral Medicine \\ University of Massachusetts Medical School \\ Danielle.JakeSchoffman@umassmed.edu
}

\author{
Stephanie Carreiro \\ Emergency Medicine and Medical Toxicology \\ University of Massachusetts Medical School \\ Stephanie.Carreiro@umassmemorial.org \\ Nathalie Nader \\ University of Massachusetts Medical School \\ Nathalie.Nader@umassmed.edu \\ Sherry Pagoto \\ Division of Preventive and Behavioral Medicine \\ University of Massachusetts Medical School \\ Sherry.Pagoto@umassmed.edu
}

\begin{abstract}
Increased access and use of social media on smartphones and tablets have changed interpersonal communication styles. Because of the ease of social media access and the ability to reach a large number of individuals, social media is an ever more important modality that connects individuals. Importantly, adolescents have adopted social media platforms to discuss issues related to mental health. There is little existing data regarding how adolescents who are depressed or suicidal use social media prior to treatment in the emergency department (ED) for medical care of their psychiatric illness. In this paper, we present formative evidence of social media behaviors in 29 adolescents seeking emergency care for depression or suicidal ideation. Participants were surveyed regarding social media use and motivations to post content regarding depression, death or dying. Among the participants who allowed the research team to view their social media accounts, 40\% $(n=6)$ posted content related to depression, death or dying, while 20\% $(n=3)$ wrote that they felt depressed and $13.3 \%(n=2)$ posted that they wanted to die. Qualitative discussions with participants provided
\end{abstract}

description of reasons for posting content on social media about depression, death and dying, or reasons that individuals refrained from posting online. Despite methodological and technical challenges in research, social media may prove be valuable in detection and intervention of adolescents who are depressed and contemplating suicide.

\section{Introduction}

Social media platforms have changed interpersonal communication. The combination of nearly ubiquitous smartphone access, and the transition of social media platforms onto smartphones has resulted in daily use among teens [8]. Unique to the current generation is the use of social media from an early age. A 2012 survey showed $95 \%$ of $12-17$ year olds regularly access the internet, and $81 \%$ of them use some form of social media [10]. As of $2015,91 \%$ of teens report going online at least once daily, with $24 \%$ reporting they are online "almost constantly" [10]. In compliance with the Children's Online Privacy Protection Act (COPPA), social media platforms require a minimal age of 13 to register for an account. There is limited research in social media use in 
younger children, however there is anecdotal evidence that children younger than 13 are using social media.

The popularity of social media has led to its use as an outlet to discuss mental health and more specifically, depression and self-harm, among adolescents for a variety of reasons [13]. First, adolescents may feel more comfortable reaching out through social media than traditional outlets during vulnerable periods of acute depression and desire to inflict self-harm. In addition, anonymity in social media platforms allows adolescents to discuss and learn about methods of self-harm without the knowledge of parents and close friends.

Among adolescents, suicide is the second leading cause of death in the US with one young person committing suicide every two hours. Data from the Centers for Disease Control (CDC) indicates that youth suicide rates have increased by $24 \%$ between 1999-2014 [7]. Children as young as age 5 are at risk for suicide. It may be difficult for medical providers to predict suicide; research into risk factors and suicide rating scales have not revealed consistently definitive methods to determine who will attempt or complete suicide [12]. Anecdotal aggregate data from our group practice has revealed themes of individuals posting about thoughts or intents of self-harm on popular social media sites. Additionally, multiple cases in the national headlines have highlighted individuals posting suicidal content or notes on social media and then following through with a completed suicide. Recent evidence suggests that adolescents use social media sites to display comments about their suicidal thoughts, behaviors, and attempts [6]. Methods to identify, access and intervene upon at-risk adolescents and those with active suicidal intent are limited; social media may provide an opportunity to detect periods when adolescents are in a vulnerable mental state.

A recent study in China tested the possibility of building a suicide dictionary using the social media service provider Sina Weibo; researchers were able to build a dictionary that significantly correlated with expert ratings in terms of both detecting suicidal expression and evaluating individual suicide risk [11].

In the US, numerous social media platforms are available, with Facebook, Instagram, Twitter, and Snapchat being the most frequently used by teens (1317 years old) and young adults (18-29 years old) [5, 10]. Among these platforms Facebook and Twitter allow for posting text, links to websites, images, and videos, Instagram and Snapchat are restricted to posting images and videos [1-4]. All four platforms have embedded chat/messaging functions to facilitate private conversations between users. Accounts may be private (where users can post content that is only shared with their "friends" or other users who they have authorized to "follow" them) or where anyone can see posted content without special permissions. Unlike Facebook, Instagram, and Twitter, where content is posted permanently (unless specifically deleted by the user), Snapchat is designed as an "autodelete" messaging app and images and videos (10second recording limit) that users post as their "story" are available for 24 hours and then disappear.

To our knowledge, this is the first paper to systematically review and describe public and private social media postings to assess whether certain language, content, and conversations might be indicative impending suicide attempt. Here, we describe the use of social media in adolescents presenting for emergency care with psychiatric complaints or suicide attempts. We also describe methodological and technical challenges for investigators who seek to conduct social media based studies in adolescents with mental health.

\section{Materials and Methods}

The current study investigated adolescents who presented to a hospital for acute medical and/or psychiatric care for suicidal thoughts or attempts. Briefly, participants were consented and enrolled in the hospital after emergency department care was provided. Participants were first administered a survey regarding social media posting habits and the use of social media to convey topics related to mental health. Following the survey, participants were queried in a qualitative discussion regarding their opinions of posting content about their own mental health on social media. Participants were separately consented to provide the research team access to their social media accounts. A combination of the administered survey and social media data was collated and analyzed to describe the participant use of social media prior to ED presentation. The study took place in an urban academic medical center in Massachusetts. The study was approved by our institutional review board.

\subsection{Eligibility and Consent}

English speaking patients, ages 9-22, presenting to the hospital with a self-harm related complaint (i.e. suicidal ideation, intentional overdose) were eligible for this study. Patients were enrolled on a convenience basis over a six month period. Eligible patients were identified through query of the electronic medical record (EMR) for presenting complaints of mental health (eg. depression or seeking psychiatric care) or suicide ideation or attempt. Eligible participants were 
approached by a member of the research team regarding study participation. We obtained written consent by participants' parent/guardian in addition to written consent by participants if they were minors (under 18 years of age). This study utilized two consents, first for participation in a survey, second for granting the research team permission to access their personal social media accounts. Individuals who enrolled in the survey portion were also asked to grant the research team permission to review their social media postings, but this was not required to participate in the study.

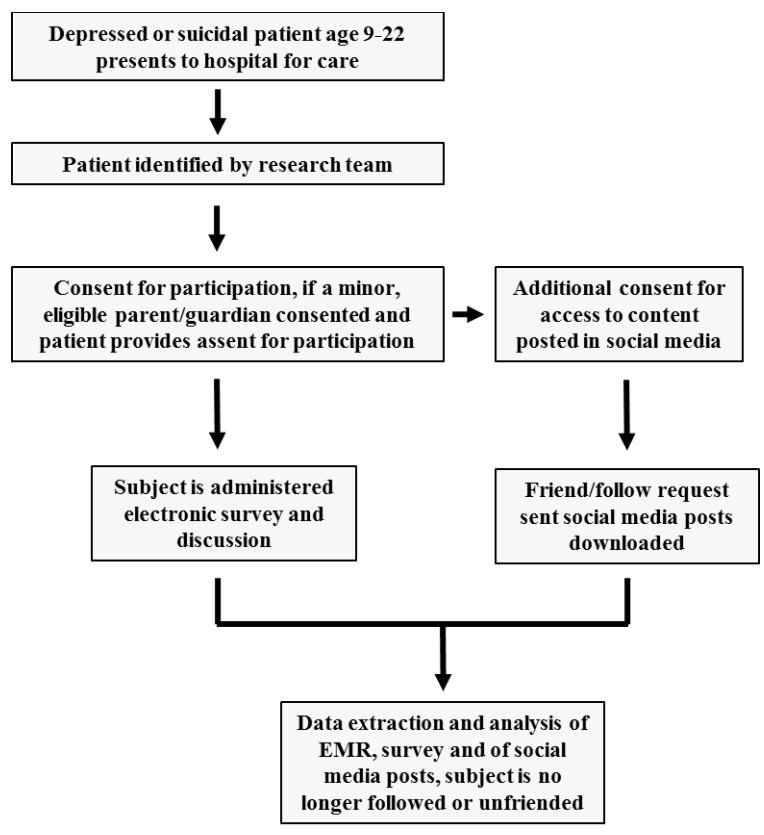

Figure 1: Overall Flow of SoMe Study

\subsection{Data Collection}

Data was collected in Research Electronic Data Capture (REDCap). REDCap is a secure, web-based application for building and managing online surveys and databases [9]. Demographic data was extracted from the participants' EMR.

The survey asked the participant to answer questions regarding social media habits, including the type(s) of social media platforms accessed, and the frequency of use. Participants were asked to answered a series of questions regarding the use of social media to communicate mental health topics, such as if they posted any content about depression, consideration of harming themselves or statements about dying. The survey asked participants to indicate how frequency content was posted content, how soon prior to the hospitalization content was posted, and what platforms were used to post content.

The survey was followed by a brief qualitative discussion. Participants were asked an open-ended questions regarding their opinion on posting content about their own mental health on social media. Participant responses were documented verbatim by a member of the research team.

Participants who used Facebook, Instagram or Twitter who consented to allow the research team to review their accounts provided their account username. For social media platforms that are not public, a friend or follow request was sent using a dedicated study account on the corresponding social media platform. If the participant did not accept the request, they were ineligible for this portion of the study. Three months following consent, participant accounts were no longer accessed. The dedicated study social media accounts had security settings that prohibited participants from posting content to the study account and prevented participants from viewing who the study accounts were following or "friends" with.

Social media posts through one month prior to review to the time of consent were collected and saved in a secure location. This timeframe was selected to evaluate for warning signs just prior to their ED presentation. Content posted after the individuals were enrolled was not included. Posts were scrubbed of all identifying data- this included any information about the participant and the participant's account "friends".

\subsection{Data Extraction and Analysis}

Demographic information and survey data are reported in a descriptive manner.

Social media posts including, but not limited to text, emoticons, photos, videos and memes were reviewed by two trained independent coders. Each coder reviewed all social media posts, and categorized posts related to depression, death or dying, or selfinjurious attempts. All discrepancies between coders were re-reviewed by both coders and a third reviewer (JC).

Qualitative interviews with patients were analyzed in a similar fashion. Discussions with participants were documented by a member of the research team. These were reviewed by a trained research staff member and coded into three themes: motivation for posting, reasons to refrain from posting, other behaviors. 


\section{Results}

During the study period, a total of 37 individuals met eligibility criteria and were approached regarding enrollment. $78.4 \%(n=29)$ consented to participate in the study. Figure 2 outlines all eligible patients that were approached and outlines the reasons participants did not enroll in the study.

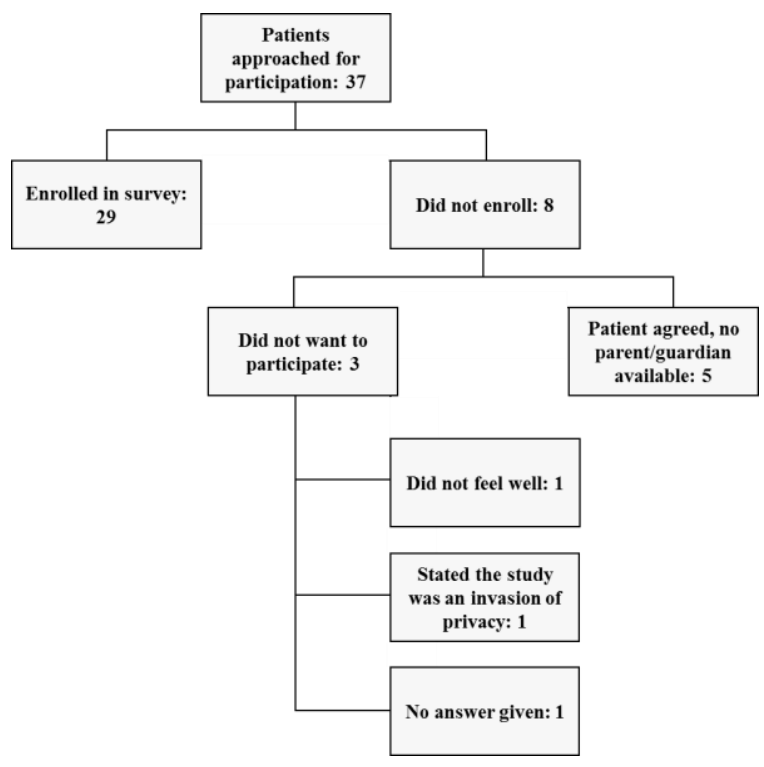

Figure 2: Study Participant Enrollment

\subsection{Participant Demographics}

The majority of enrolled participants were white females. Participant gender, age, and racial characteristics are shown in Table 1.

Table 1: Participant demographic characteristics

\begin{tabular}{|c|c|}
\hline \multicolumn{2}{|l|}{ Gender } \\
\hline Female & $75.9 \%(n=22)$ \\
\hline Male & $24.1 \%(n=7)$ \\
\hline Average age & 17.5 (range13-22) \\
\hline \multicolumn{2}{|l|}{ Race } \\
\hline White & $86 . \%(n=25)$ \\
\hline $\begin{array}{l}\text { Black or African } \\
\text { American }\end{array}$ & $3.4 \%(\mathrm{n}=1)$ \\
\hline Hispanic or Latino & $10.3 \%(n=3)$ \\
\hline
\end{tabular}

\subsection{Survey}

Participants were asked to report the electronic devices they had access to, and their primary mode of communication, results are reported in Table 2.

\section{Table 2: Communication technology}

\begin{tabular}{|l|l|}
\hline $\begin{array}{l}\text { Access to a } \\
\text { computer }\end{array}$ & Yes: 86.2\% $(\mathrm{n}=25)$ \\
\hline Personal cell phone & Yes: 89.7\% $(\mathrm{n}=26)$ \\
\hline $\begin{array}{l}\text { Device most often } \\
\text { used for social } \\
\text { communications }\end{array}$ & $\begin{array}{l}\text { Cell phone 93.1\% }(\mathrm{n}=27) \\
\text { Tablet or iPad 6.9\% }(\mathrm{n}=2)\end{array}$ \\
\hline
\end{tabular}

Participants reported the total number and specific names of unique social media platforms or apps they used on a daily basis to communicate with others. Results are shown in Figures 3 and 4.

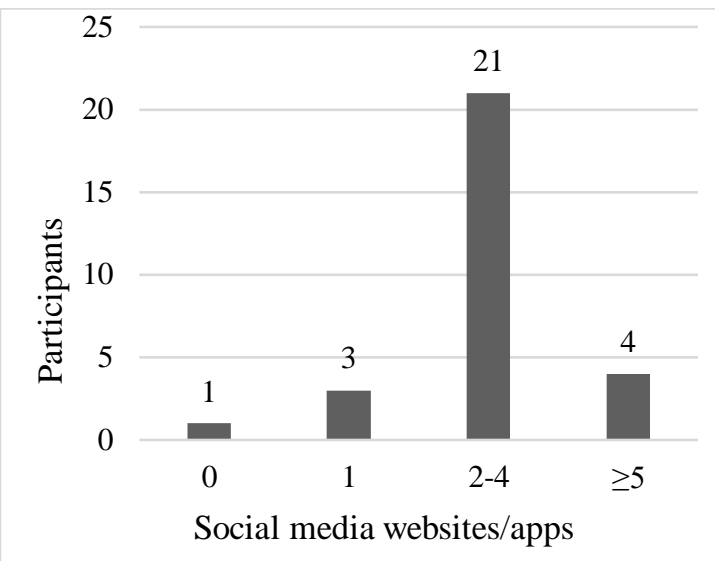

Figure 3. Number of unique social media platforms used daily

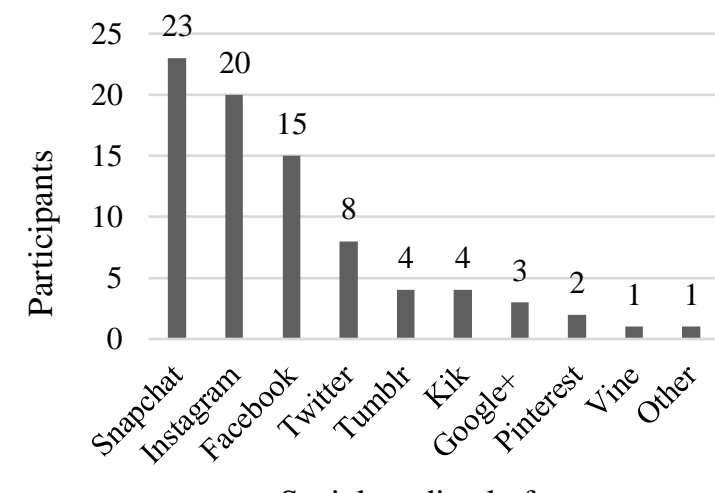

Social media platform

Figure 4. Social media platforms used by participants on a daily basis 


\subsection{Social Media}

All enrolled participants consented to the survey $(n=29)$. Of these, $79.3 \%(n=23)$ also signed consent to allow the research team access to review their social media content. In the subset of participants who did not consent, three did not have parent/guardian permission (parent/guardian reported dislike for social media). The remaining three reported they did not have a social media account or did not post to social media. Ten participants provided access to their Instagram accounts, with nine accepting the research team's request to follow their account. Nine provided the research team with their Facebook account information, but only six accepted our friend request, and four participants consented to, and granted us permission to follow their Twitter accounts. Thus, of the 23 people who consented to the social media component, we were able to review posts for 19 $(82.6 \%)$ participants. Upon review, we found that four participants did not post any content in the month leading up to enrollment in the study. For the remaining 15 participants, $40 \%(n=6)$ posted content related to depression, death or dying. $13.3 \%(n=2)$ participants specifically wrote in a post that they wanted to die, and $20 \%(n=3)$ wrote that they felt depressed. Total number of posts and content related to depression, death and dying by type of content are shown in Table 3.

Table 3: Content of Post by Type

\begin{tabular}{|l|c|c|}
\hline & $\begin{array}{l}\text { Total } \\
\text { Posts }\end{array}$ & $\begin{array}{l}\text { Posts about } \\
\text { depression, } \\
\text { death or dying }\end{array}$ \\
\hline Pictures & 376 & 6 \\
\hline Videos & 161 & 4 \\
\hline Written & 296 & 19 \\
\hline Total & $\mathbf{8 3 3}$ & $\mathbf{2 9}$ \\
\hline
\end{tabular}

\subsection{Semi-Structured Discussions with Participants}

During a qualitative interview, participants further elaborated on social media use and content posted. Themes identified in these discussions included: (1) Motivation to post (2) Reasons to refrain from posting and (3) Other behaviors identified.

Motivation to post: participants reporting posting on social media because it is preferred over interpersonal discussions. One participant noted she does not like expressing her emotions in person, and posted "that kind of stuff" (i.e. feelings of sadness or depression) on social media. Another participant described that she posts on an account that only her closest friends were allowed access. She reported that by posting this content, it "kind of" helps her feel better. Another participant who posted anonymously stated he was able to find "other people who understand."

Reasons to refrain from posting: Participants reported they did not post because they did not want others to know about their depression and suicidality. Specific comments included a participant who reported she was "a "private person", another participant noted that he did not post about his feelings on social media because his family members are "friends" with his account; this he did not want family to see his posts. Another participant stated the reason for not using popular platforms was because he felt that he did not fit in with other students in his high school; this participant identified as gay and felt that "everyone at his school was homophobic."

Other behaviors identified: the above participant reported using dating apps instead of popular social media sites to remain anonymous. Participants admitted to having multiple accounts within and across platforms with varied content posted. One participant reported that she did not post content on her primary accounts; however she did post to her "Finsta" account (Finsta is known to be a "fake" Instagram account.) Another participant described posting to four separate Facebook accounts. She declined to specify what was posted to, or who was able view each account, but indicated that there was one account where she had posted more content related to her feelings. Another participant reported that she had already deleted previously posted content from her account.

\section{Discussion}

Prior to an individual attempting suicide, a person may project warning signs. Our study demonstrated that of the individuals who posted content on social media, nearly half of them posted about depression, death or dying, with two of them specifically posting about the desire to die. This however may be an underrepresentation of the actual number posting this type of content, as participant reported using multiple social media accounts but may have only given us access to certain accounts that did not contain about depression, dying or dying.

Qualitative discussions with participants revealed motivations for posting content within social media platforms or their reasons for refraining from posting. 
Some individuals described posting online enabled them to maintain anonymity, and found posting helpful. Others reported they refrained from posting because they did not want their online network to know about their feelings.

This data is significant because we report that individuals may post intent or actual suicide attempts on social media in the month prior to their presentation to the hospital. It also helps us to understand the behaviors related to decisions to post content to social media. We demonstrate in our study population that adolescents are accessing social media on smartphones multiple times a day and platforms most commonly used in our study are consistent with national trends [10]. Therefore, this data suggests that interventions addressing suicidal intent or impending events targeted to adolescents may be delivered through social media platforms.

Although social media content is generally public, we found that relatively private events (i.e. worsening depression, suicidal intent, and suicide attempts) were displayed on social media platforms. We also found that adolescent social media profiles are complex; participants tend to utilize multiple social media platforms and may have multiple linked or even unlinked accounts in order to anonymously compose posts about suicide. Interestingly, given the public nature of social media, adolescents have developed methods to anonymize personal posts through "fake" unlinked Instragram accounts (eg. Finsta), or multiple unlinked Facebook accounts. Adolescents may, therefore, seem happy and stable on their "public" profiles while they post important indicators of worsening mental health or even suicidal ideation on alternative accounts. Additionally, adolescents may be using alternative social media platform that may be overlooked. For example, one participant described the use of popular dating apps to express worsening mental health. This poses an important consideration in clinicians seeking to develop social media interventions and access adolescents on social media. Interventions that respond to at-risk adolescents through social media should consider methods to securely access not only known social media accounts, but also "fake" and unlinked accounts.

While websites and blogs exist for anonymous dialogue of suicide and depression, adolescents use social media platforms to post and discuss worsening mental health and suicidal ideation. Understanding the transition point from contemplation to actual attempt based on either frequency or category of social media posts may allow therapists, families and physicians to detect early evidence of deteriorating mental health and intervene before individuals present to the ED. Our study shows that adolescents spend time on various social media platforms. Connecting with adolescents on these variable platforms may help augment existing psychotherapy or pharmacotherapy.

\subsection{Limitations of Social Media Research}

We found that despite individuals consenting to allow the research team accessing their data for research purposes, not all individuals approved our request on social media. While do not know the reason behind this, we found that some participants were transferred to psychiatric facilities without access to internet or cell phones, parents reported banning using social media; in other cases the individual may have made the decision to decline participation in this aspect of the study.

Gaining access to all content posted is a challenge due to the use of multiple platforms or accounts within a platform. The popular platform, Snapchat allows users to exchange photographs or videos that will then disappear after a maximum of ten second; the lack of permanency associated with disappearing content may be appealing to this group of adolescents posting about depression and suicidal ideation. Researchers conducting studies using this popular platform would be unable available for review. In addition, individuals may post content on any platform, but may remove it at any time.

Finally, our sample size was relatively small and does not include a similar matched comparison group of adolescents who presented to the hospital for reasons other than mental health treatment.

\subsection{Strengths and Study Limitations}

Noted strengths of the study include that the study population is a real-world sample of adolescents in crisis. Social media content related to depression, death and dying was not subject to a reporting bias; content included in the study was posted prior to participant enrollment and was therefore genuine and not influenced by the study.

This study also had limitations. First, the study was conducted at a single academic hospital. Although the hospital is major referral center for pediatric emergencies, the demographics of our study population may not reflect individuals that present to hospitals for across the country.

Second, we gained incomplete access to participants' social media content. Participants may have chosen only to share with us accounts are most public and do not contain any content about depression or suicide. 


\subsection{Future Directions}

In our future studies, we would like to explore individuals' social media postings, with a focus on content; this would include not only mentions of depression and dying, but frequency of content and sentiment of posts in the time leading up to their hospital presentation for treatment. We plan to investigate further by identifying differences in individuals based on the severity of their depression or suicide attempt.

\section{Conclusion}

Social media provides a rich data source for behavioral health researchers. There has been limited research on depressed and suicidal adolescents' use of social media. This study demonstrates the feasibility accessing public and private social media accounts for depressed and suicidal adolescents seeking acute medical and psychiatric care. We show that patients are willing to provide access to public and private accounts. However, due to the wide variety of social media platforms, users posting on multiple sites daily, and the practice of using multiple accounts within platforms, it is difficult to capture an individual's complete posting history on social media for research purposes. Continued research will help improve methods for social medial research in depressed and suicidal adolescents.

\section{References}

$\begin{array}{ll}\text { 1. } & \text { https://twitter.com/. } \\ \text { 2. } & \text { https://www.facebook.com/. } \\ \text { 4. } & \text { https://www.instagram.com/. } \\ 5 . \quad & \text { https://www.snapchat.com/. } \\ \text { Anderson, M.P.R.C., January, 2016, "Parents, } \\ \text { Teens and Digital Monitoring." Available at } \\ \text { http://www.pewinternet.org/2016/01/07/parents- } \\ \text { teens-and-digital-monitoring/. } \\ \text { Cash SJ, T.M., Peck SN, Ferrell JZ, Bridge JA. } \\ \text { Adolescent suicide statements on MySpace. } \\ \text { Cyberpsychol Behav Soc Netw. } 2013 \\ \text { Mar;16(3):166-74. } \\ \text { Curtin SC, W.M., Hedegaard H. Increase in } \\ \text { suicide in the United States, 1999-2014. NCHS } \\ \text { data brief, no 241. Hyattsville, MD: National } \\ \text { Center for Health Statistics. 2016. } \\ \text { Felt, L.J.R., M. B. (2016). Technology addiction: } \\ \text { Concern, controversy, and finding balance. and } \\ \text { C.C.S.M. San Francisco. } \\ \text { Harris PA. Research Electronic Data Capture } \\ \text { (REDCap) - planning, c.a.m.d.f.c.a.t.r.B.B.S.A., } \\ \text { Research Electronic Data Capture (REDCap) - } \\ \text { planning, collecting and managing data for }\end{array}$

clinical and translational research. BMC Bioinformatics 2012;13(Suppl 12):A15.

10. Lenhart, A.T., Social Media \& Technology Overview 2015, Pew Research Center. Available at: http://www.pewinternet.org/2015/04/09/teenssocial-media-technology-2015/.

11. Lv, M., et al., Creating a Chinese suicide dictionary for identifying suicide risk on social media. PeerJ, 2015. 3: p. e1455.

12. Muzina, D.J., What physicians can do to prevent suicide. Cleve Clin J Med, 2004. 71(3): p. 242-50.

13. Pantic, I., Online social networking and mental health. Cyberpsychol Behav Soc Netw, 2014. 17(10): p. 652-7. 